\title{
Quality of life in euthymic patients with unipolar major depressive disorder and bipolar disorder
}

This article was published in the following Dove Press journal:

Neuropsychiatric Disease and Treatment

\author{
Qijing Bo ${ }^{1-4, *}$ \\ Lu Tian ${ }^{1-4, *}$ \\ Feng $\mathrm{Li}^{1-4}$ \\ Zhen Maol-4 \\ Zhimin Wang ${ }^{\mathrm{I}-4}$ \\ Xin $\mathrm{Ma}^{\mathrm{I}-4}$ \\ Chuanyue Wang ${ }^{1-4}$
}

'The National Clinical Research Center for Mental Disorders, Beijing Anding Hospital, Capital Medical University, Beijing 100088, People's Republic of China; ${ }^{2}$ Beijing Key Laboratory of Mental Disorders, Beijing Anding Hospital, Capital Medical University, Beijing 100088, People's Republic of China; ${ }^{3}$ Beijing Institute for Brain Disorders Center of Schizophrenia, Beijing Anding Hospital, Capital Medical University, Beijing 100088, People's Republic of China; ${ }^{4}$ Advanced Innovation Center for Human Brain Protection, Capital Medical University, Beijing 100069, People's Republic of China

*These authors contributed equally to this work
Correspondence: Qijing Bo Beijing Anding Hospital, Capital Medical University, No.5 Ankang Lane, Dewai Avenue, Xicheng District, Beijing 100088, People's Republic of China $\mathrm{Tel} / \mathrm{Fax}+861058303119$ Email bqj718@।63.com

\begin{abstract}
Objective: This study aimed to compare quality of life (QOL) between patients with major depressive disorder (MDD) in remission and patients with bipolar disorder (BD) in remission, and to explore the relationship between QOL and demographic, clinical, and cognitive variables.

Methods: This study included 49 euthymic patients with MDD, 59 euthymic patients with BD, and 52 healthy controls (HC). The 17-item Hamilton Depression Rating Scale (HAMD17), Hamilton Anxiety Rating Scale (HAMA), and Young Mania Rating Scale (YMRS) were used to assess symptoms of depression, anxiety, and mania respectively. QOL was assessed with the Chinese version of the World Health Organization Quality of Life Scale Brief (WHOQOL-BREF). Cognitive function was assessed with the repeated neuropsychological assessment scale (RBANS).

Results: Compared with HC, patients with MDD had lower overall and subdomain scores (except ENVIR) on the WHOQOL-BREF $(p<0.05)$. The BD group had decreased overall WHOQOL-BREF scores and decreased PHYS and SOCIL subdomain scores $(p<0.05)$. PSYCH scores were lower in patients with MDD, compared with patients with BD $(p<0.05)$. Among patients with MDD, HAMD score was negatively correlated with all domains on the WHOQOL-BREF. Marital status was associated with an increase in subdomain scores on the PSYCH and ENVIR subdomains. In the BD group, attention on the RBANS correlated negatively with PSYCH score; age correlated negatively with SOCIL.

Conclusions: QOL of patients with MDD and BD in remission is inferior to that of the normal population. QOL among MDD is inferior to that among BD. Marital status was associated with increased QOL among MDD, but not among BD. Residual symptoms related to depression or anxiety decreased QOL in both MDD and BD. More attention should be paid to the QOL of patients with mood disorders, especially MDD, even during euthymic periods.
\end{abstract}

Keywords: major depressive disorder, bipolar disorder, quality of life, euthymic, cognitive function

\section{Introduction}

Mood disorders, also called affective disorders, are characterized by disturbances in mood, disposition, and behavior, or fluctuations in mood from a baseline, that affect an individual's participation in the activities of daily life. There are two main mood disorder diagnoses: major depressive disorder (MDD) and bipolar disorder (BD). The two diagnoses differ substantially. Patients with unipolar depression are merely involved in a low mood state, whereas, patients with BD suffer from both low and high mood. ${ }^{1}$ Patients with MDD or BD may be feeling abnormally good or sad for a long period of time and without any apparent reason. 
Quality of life (QOL), defined by the World Health Organization (WHO) as "individuals" perceptions of their position in life in the context of the culture and value systems in which they live and in relation to their goals, expectations, standards and concerns", is a broad concept involving physical health, psychological state, personal beliefs, level of independence, social relationships, and living environment. ${ }^{2}$ As several studies have shown that both somatic diseases and mental diseases impair QOL, the measure has been used to assess the effectiveness of mental health services. ${ }^{3,4}$ QOL is impaired in MDD and in $\mathrm{BD} .{ }^{1,5,6}$ Increasing evidence suggests that QOL is an important target of well-being, and an important index for patient health. ${ }^{7}$ Measurements of QOL have even been recommended as a measure of treatment efficacy and recurrence of depression. ${ }^{8,9}$

There has been a relatively large body of research addressing QOL in patients with mood disorders. It has been demonstrated that QOL is impaired during acute episodes of BD and MDD. One previous study investigated QOL among patients with chronic BD and MDD. ${ }^{10}$ Another study used scores on the MOS 20ITEM short form (SF-20) to demonstrate that patients with BD in clinical remission had reduced QOL. ${ }^{11}$ Clinically euthymic patients with $\mathrm{BD}$ continued to show impaired QOL. ${ }^{12}$ Another study confirmed that patients in the stable phase of BD showed lower QOL. ${ }^{13}$ Several authors have reported that QOL is not reduced in patients with MDD in remission. ${ }^{9}$ However, conflicting reports have been published. One recent study showed that decreased QOL persists during stable stages of MDD. ${ }^{14}$ This impairment in QOL is attributed to residual depressive and cognitive symptoms. ${ }^{15}$ The main study hypothesis is that QOL may be impaired in patients with mood disorders, who are in remission. The manifestations of this impairment may be affected by demographic characteristics, clinical symptoms, and cognitive function.

However, few studies have compared QOL between euthymic patients with MDD and euthymic patients with BD. The current study aimed to study both MDD and $\mathrm{BD}$, compare $\mathrm{QOL}$ of euthymic patients with MDD to that with $\mathrm{BD}$, and to probe the relationships between QOL and demographic characteristics, clinical symptoms and cognitive function, using World Health Organization Quality of Life Scale Brief (WHOQOLBREF).

\section{Materials and methods}

\section{Participants and setting}

This is a cross-sectional study conducted at Beijing Anding Hospital, Capital Medical University, during the period from September 2014 to September 2016. The patients were enrolled through the outpatient department and required to meet the inclusion criteria: (1) age between 18 and 55 years; (2) BD type 1 or MDD according to DSM-IV, currently in remission; (3) remission defined as HAMD-17 total scores $\leq 7$, YMRS total scores $\leq 6$; (4) Modified electra convulsive therapy (MECT) was not accepted in the previous three months; and (5) $\geq 9$ years of formal education. The exclusion criteria were (1) history or current significant medical or neurological conditions; (2) history or current significant drug/alcohol abuse; (3) pregnant women; and (4) severe suicidal thoughts or suicide attempt. The healthy controls (HC) were enrolled through social recruitment by recruitment advertisement from the community followed by an interview and meet the inclusion criteria: (1) age between 18 and 55 years; (2) inconformity any psychiatric disorders diagnostic criteria for DSM-IV; and (3) no related family history. Exclusion criteria were (1) history or current significant medical or neurological condition; (2) history of head trauma or unconsciousness lasting $>1 \mathrm{hr}$; (3) history or current significant drug/alcohol abuse; and (4) pregnancy.

The clinical research ethics committees of Beijing Anding Hospital approved the study protocol. Each participant provided his or her written informed consent.

\section{Assessments}

Each participant's socio-demographic data were collected with a questionnaire designed for the study. All participants were diagnosed with Structured Clinical Interview for DSM-IV-TR Axis I Disorder-patient Edition (SCID-I/ P). ${ }^{16,17}$ The 17-item Hamilton Depression Rating Scale (HAMD-17), ${ }^{18}$ Hamilton Anxiety Rating Scale (HAMA), ${ }^{19}$ and Young Mania Rating Scale (YMRS) ${ }^{20}$ were used to assess depressive, anxiety-related, and manic symptoms, respectively. Therefore, QOL was assessed with Chinese version of WHOQOL-BREF, which had four subdomains: physical health (PHYS); psychological health (PSYCH); social relationship (SOCIL) and environment (ENVIR), the total score was a comprehensive subjective score of overall QOL. ${ }^{21}$ 
Neurocognitive functioning was assessed with the Repeatable Battery for The Assessment of Neuropsychological Status (RBANS), ${ }^{22,23}$ which has 5 domains (12 tests): attention (digital span, coding); language (picture naming, semantic fluency); visuospatial/constructional (figure copy, line orientation); immediate memory (list learning, story memory); and delayed memory (list recall, list recognition, story recall, figure recall). The raw scores of each domain were summed to yield a total score representing overall level of cognitive function. Executive function was assessed with the Stroop Color Test (SCT). The SCT consists of three conditions: word, color, and interference. ${ }^{24}$ Under one set of conditions, total time required to perform the task is used as a measure of performance. For the word task (Sword time), the participant is asked to read out the names of various color words, written in black ink. For the color task (Scolor time), the participant is asked to state the color of a block of colored ink. For the interference task, the participant must determine the correct word, despite conflicting information, in terms of letter color (Dword time) and then must determine the correct color, despite conflicting information, in terms of word meaning (Dcolor time).

\section{Procedure}

Sociodemographic data were obtained for all participants included in the study. Eligible participants continued to complete symptom scales, WHOQOL self-assessment scales, and neurocognitive functions assessment. The selfdesigned questionnaire used a unified instruction and fill in a form. The subjects were completed independently. Clinical assessments were completed by trained psychiatrists. Inter-rater reliability remained within acceptable limits.

\section{Statistical analysis}

Data were entered using Epidata software version 3.1 and were analyzed using SPSS 25.0 for Windows (SPSS, Inc., Chicago, IL, USA). The one-way ANOVA was used to compare the demographic, clinical, QOL, and neuropsychological tests among the three groups, Bonferroni correction was used to measure multiple group differences. ${ }^{25}$ QOL and cognitive function were subjected to analysis of covariance with significant demographic variables. ChiSquare test was used to analyze gender difference, marital status, and current drugs using among the three groups. Pearson or Spearman rank correlation analysis was performed to measure the associations of QOL with socio- demographic and clinical characteristics and performance on other neurocognitive tests. Stepwise Multiple Regression Analysis was used to identify factors that were independently associated with QOL. For all analyses, the level of statistical significance was set at $p<0.05$.

\section{Results}

\section{Demographic and clinical characteristics}

A total of 49 patients with MDD and 59 patients with BD in remission, $52 \mathrm{HCs}$ who met the study criteria entered the study. The three groups did not differ with respect to gender, educational level, marital status, or current use of drugs. Members of the MDD group were significantly older than those of the other two groups $(\mathrm{F}=8.96, p<0.001)$. In the other hand, the patient groups did not differ with respect to duration of illness, but the age at onset of illness and recurrence times. In addition, patients showed significantly higher scores of HAMD and HAMA than HC. Patients with $\mathrm{BD}$ in remission scored higher on the YMRS than did patients with MDD or HC. Table1 shows the demographics characteristic and the score of HAMD-17, HAMA, YMRS.

\section{Comparison of QOL and cognitive function among MDD and BD patients and $\mathrm{HCs}$}

Compared with HC, the MDD group had lower scores for the total WHOQOL-BREF and its subdomains (except ENVIR) $(p<0.05)$. The BD group had decreased scores for WHOQOL-BREF overall, as well as the PHYS and SOCIL subdomains $(p<0.05)$. MDD patients scored significantly lower on PSYCH than did BD patients $(p<0.05)$. Figure 1 presents differences in QOL domain scores among the groups. Because age differed significantly among groups, we used age as a covariate for analysis of QOL. This had no impact on the results.

Performance differed among the three groups only for tests of delayed memory in the RBANS subdomain $(p<0.05)$. However, after Bonferroni correction, this difference was not significant. Compared with the HC group, the BD group had increased Scolor time on SCT $(p<0.05)$. When age was used as a covariate, the significance of the result remained unchanged.

\section{Variables associated with QOL among MDD and BD patients}

Table 2 shows the correlations between demographic and clinical characteristics, neurocognitive function, and 
Table I Demographics characteristic and QOL scores

\begin{tabular}{|c|c|c|c|c|c|c|c|c|c|}
\hline \multirow[t]{2}{*}{ Characteristics } & \multicolumn{2}{|c|}{ MDD (n=49) } & \multicolumn{2}{|c|}{ BD $(n=59)$} & \multicolumn{2}{|c|}{$H C(n=52)$} & \multicolumn{2}{|c|}{ Statistics } & \multirow[t]{2}{*}{ Post Hoc Analysis } \\
\hline & Mean & SD & Mean & SD & Mean & SD & $\mathbf{F}$ & $p$ & \\
\hline Age (years) & 37.59 & 10.08 & 30.31 & 10.23 & 36.92 & 10.23 & 8.96 & $<0.001$ & $\mathrm{BD}<\mathrm{MDD}, \mathrm{HC}$ \\
\hline Education (years) & 13.94 & 3.45 & 13.59 & 3.16 & 13.48 & 2.81 & 0.29 & 0.748 & - \\
\hline Duration of illness (months) ${ }^{\mathrm{a}}$ & 86.49 & 83.71 & 81.07 & 84.89 & - & - & 0.11 & 0.740 & - \\
\hline Age at onset ${ }^{\mathrm{a}}$ & 30.96 & 10.95 & 24.02 & 9.08 & - & - & 12.97 & $<0.001$ & $\mathrm{BD}<\mathrm{MDD}$ \\
\hline Recurrence times ${ }^{\mathrm{a}}$ & 2.14 & 1.28 & 3.39 & 2.19 & - & - & 12.39 & 0.001 & \\
\hline HAMD total score & 2.81 & 2.29 & 2.00 & 2.01 & 0.58 & 1.19 & 18.27 & $<0.001$ & $\mathrm{HC}<\mathrm{BD}<\mathrm{MDD}$ \\
\hline HAMA total score & 4.23 & 4.79 & 2.56 & 3.04 & 0.62 & 1.37 & 14.95 & $<0.001$ & $\mathrm{HC}<\mathrm{BD}<\mathrm{MDD}$ \\
\hline YMRS total score & 0.31 & 1.01 & 2.49 & 4.71 & 0.85 & 1.75 & 7.38 & 0.001 & $M D D, H C<B D$ \\
\hline \multicolumn{10}{|l|}{ WHOQL-BREF domains } \\
\hline PHYS & 13.34 & 2.24 & $|4.3|$ & 2.31 & 15.55 & 1.63 & 14.24 & $<0.001$ & $\mathrm{MDD}, \mathrm{BD}<\mathrm{HC}$ \\
\hline PSYCH & 13.24 & 2.31 & 14.32 & 2.37 & 15.05 & 1.98 & 8.41 & $<0.001$ & $M D D<B D, H C$ \\
\hline SOCIL & 12.98 & 2.61 & 13.56 & 2.56 & 14.92 & 1.92 & 8.93 & $<0.001$ & $\mathrm{MDD}, \mathrm{BD}<\mathrm{HC}$ \\
\hline ENVIR & 13.70 & 2.15 & 13.82 & 2.54 & 14.08 & 2.01 & 0.36 & 0.696 & - \\
\hline Total scores & 73.74 & 16.33 & 77.54 & 13.08 & 85.34 & 10.75 & 9.43 & $<0.001$ & $\mathrm{MDD}, \mathrm{BD}<\mathrm{HC}$ \\
\hline RBANS & & & & & & & & & - \\
\hline Attention & 105.89 & 15.54 & 103.00 & 14.24 & 106.87 & 13.16 & 1.08 & 0.342 & - \\
\hline Language & 93.64 & 12.74 & 89.84 & 14.32 & 95.79 & 15.35 & 2.44 & 0.090 & - \\
\hline Visuospatial & 96.67 & 15.55 & 96.60 & 15.98 & 97.92 & 17.96 & 0.11 & 0.900 & - \\
\hline Immediate memory & 87.16 & 17.18 & 83.93 & 16.31 & 88.79 & 18.89 & 1.10 & 0.337 & - \\
\hline Delayed memory & 90.73 & 16.84 & 85.37 & 13.14 & 92.92 & 14.38 & 3.13 & 0.046 & $\mathrm{BD}<\mathrm{HC}$ \\
\hline Total scores & 92.49 & 15.42 & 88.26 & 11.27 & 94.44 & 14.89 & 2.85 & 0.061 & \\
\hline \multicolumn{10}{|l|}{ SCT } \\
\hline Sword time & 17.29 & 4.16 & 17.73 & 5.95 & 16.43 & 5.05 & 0.87 & 0.422 & - \\
\hline Scolor time & 22.77 & 7.59 & 25.83 & 11.68 & 21.13 & 6.90 & 3.69 & 0.027 & - \\
\hline Dword time & 21.09 & 7.22 & 21.86 & 8.11 & 20.63 & 9.51 & 0.30 & 0.742 & - \\
\hline \multirow[t]{2}{*}{ Dcolor time } & 38.87 & 9.72 & 43.04 & 15.10 & 38.59 & 11.84 & 2.04 & 0.134 & - \\
\hline & $\mathrm{N}$ & $\%$ & $\mathrm{~N}$ & $\%$ & $N$ & $\%$ & $\chi^{\prime}$ & $p$ & \\
\hline Gender $(m e n)^{b}$ & 21 & 42.9 & 37 & 75.5 & 23 & 44.2 & 5.48 & 0.065 & - \\
\hline Married ${ }^{b}$ & 31 & 63.3 & 29 & 49.2 & 37 & 71.2 & 5.81 & 0.055 & - \\
\hline Current use of drugs ${ }^{b}$ & 7 & 14.29 & 5 & 8.48 & - & - & 0.92 & 0.339 & - \\
\hline
\end{tabular}

Notes: ${ }^{a}$ Student's $t$-test. ${ }^{b} \chi^{2}$ analysis. All other values result from analysis of variance, with Bonferroni correction used in post hoc. "Recurrence times" denotes times of relapse. Abbreviations: MDD, major depressive disorder; BD, bipolar disorders; HC, health controls. PHYS, physical health; PSYCH, Psychological health; SOCIL, social relationship; ENVIR, environment; RBANS, repeated neuropsychologicalassessment scale; SCT, Stroop Color Test; WHOQOL-BREF, World Health Organization Quality of Life Scale Brief.

different QOL domains in the group of MDD and BD. In MDD, female sex and older age were associated with higher scores on the SOCIL subdomain. Married patients scored higher on all domains related to QOL. Educational level was positively associated with SOCIL. Age at onset was positively associated with PHYS, PSYCH, and SOCIL. Residual depressive symptoms were inversely associated with scores on all subdomains related to QOL, as well as anxiety in the PHYS, SOCIL, and ENVIR subdomains. In the BD group, older age was associated with lower SOCIL scores. Recurrence times were positively associated with PHYS, PSYCH, and ENVIR. HAMD and HAMA scores were inversely associated with PSYCH. It seems that there is no significant correlation between QOL domains and neurocognitive function in MDD group, but what deserves to be mentioned is that attention deficits correlated with PSYCH and Dword time of SCT are associated with ENVIR in BD group.

Multiple regression analysis was performed to clarify the relationship between QOL and demographic and clinical characteristics. In MDD, the results showed that HAMD score was negatively correlated with all domains 


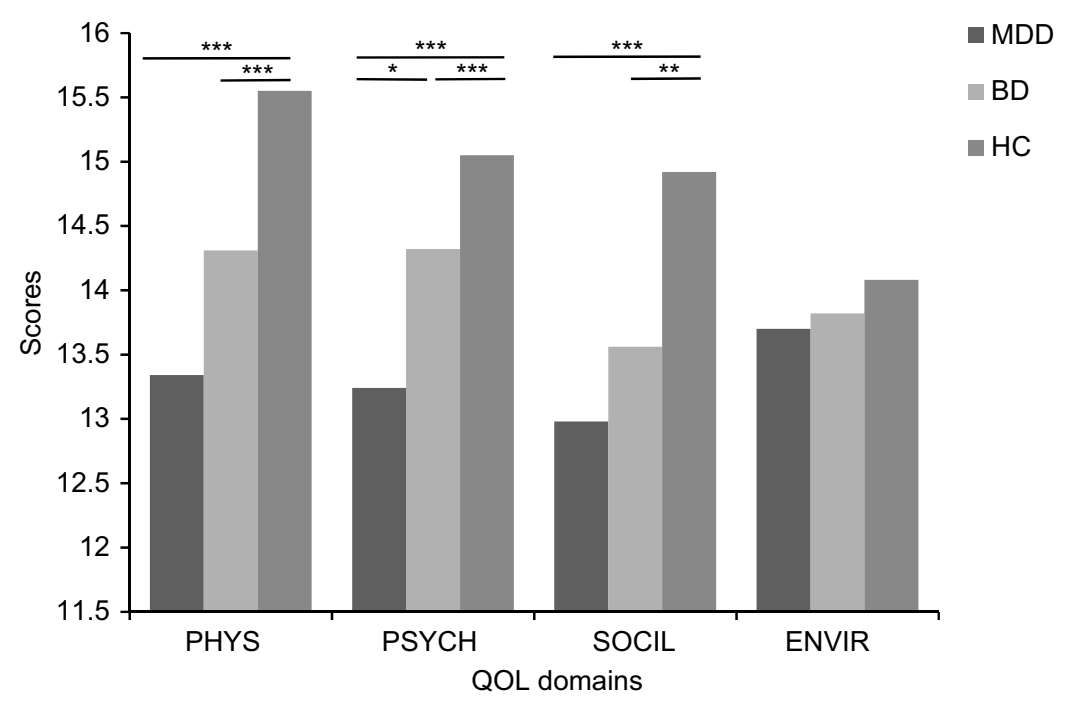

Figure I Comparison of WHOQOL-BREF domain scores between major depressive and bipolar patients, and healthy subjects. ${ }^{*} p<0.05$ ( 2 -tailed). $* * p<0.0$ I $(2$-tailed). $* * * p<0.001$ (2-tailed).

Abbreviations: MDD, major depressive disorder; BD, bipolar disorders; HC, health controls; PHYS, physical health; PSYCH, Psychological health; SOCIL, social relationship; ENVIR, environment; QOL, quality of life; WHOQOL-BREF, World Health Organization Quality of Life Scale Brief.

on the WHOQOL-BREF; marital status positively impacted scores on PSYCH and ENVIR. In BD, attention on RBANS correlated negatively with PSYCH; age correlated negatively with SOCIL. Multiple Regression Analysis results are shown in Tables 3 and 4 .

\section{Discussion}

The results suggested that the QOL of patients with the mood disorder such as MDD and BD suffered damage even in euthymic periods. QOL among MDD is inferior to that among BD. Marital status, age, HAMD score, and attention on RBANS correlated with QOL.

The results of the present study indicate that patients with MDD in remission have impaired QOL in three domains: PHYS, PSYCH, and SOCIL. The finding that marital status was associated with increased QOL in MDD is consistent with the data in the literature of the previous researches. ${ }^{26}$ Numerous studies have reported that functional recovery lags behind syndrome recovery, ${ }^{27}$ and QOL improvement lags behind clinical response. ${ }^{5}$ For instance, as previously reported, syndrome recovery does not necessarily result in a functioning comparable with that of healthy persons, which may be enduring subsyndromal symptoms. ${ }^{28}$ Some experts in the treatment of depression have advice that achieving remission of symptom should be viewed as the primary goal. $^{29}$ In the present study, patients with MDD were in remission, but the HAMD scores of these patients remained higher than those of $\mathrm{HCs}$, with negative effects in terms of QOL. Full remission is therefore needed to increase QOL and the speed of functional recovery. In addition, no domains of RBANS score and CST were associated with reduced satisfaction with QOL of MDD patients.

The results of the current study also indicate that patients with BD in remission have impaired QOL in two domains: PSYCH and SOCIL. This finding is consistent with previously published reports. ${ }^{13,30-32}$ These findings depart slightly from findings reported previously for a Chinese population, which stated that euthymic BD patients had significantly lower scores on assessments of the physical domain of QOL, compared with HC. ${ }^{33}$ Besides, QOL is being treated as a material outcome of care alongside symptom treatment in numerous studies. ${ }^{34-36}$ Furthermore, factors such as age, premorbid personality, and family support differed significantly between groups. In BD group, the QOL is negatively correlated with recurrence times, which seems unparalleled with previous study which proved that the optimal control of depressive symptoms as well as the availability of social support may enhance euthymic BD patients' well-being. $^{37}$

As for the comparison between MDD and BD groups, even controlled for age at onset and duration of illness, differences in WHOQOL-BREF domain scores were detected: the patients with MDD in remission showed lower score for PSYCH than that of BD. Obviously, there was a significant higher score of HAMD and HAMA in euthymic MDD patients than that of BD. In other word, MDD patients may fail to obtain full remission, which is defined as almost full recovery of depression symptoms to the level of a person without depression. ${ }^{27,38}$ 
Table 2 Correlations between sociodemographic, clinical characteristics, and neurocognitive functions, and WHOQLBREF domain scores in euthymic MDD and BD patients

\begin{tabular}{|c|c|c|c|c|c|c|c|c|}
\hline \multirow[t]{3}{*}{ Patients $(n=49)$} & \multicolumn{8}{|c|}{ QOL domains } \\
\hline & \multicolumn{2}{|c|}{ Physical } & \multicolumn{2}{|c|}{ Psychological } & \multicolumn{2}{|l|}{ Social } & \multicolumn{2}{|c|}{ Environmental } \\
\hline & MDD & BD & MDD & BD & MDD & BD & MDD & BD \\
\hline Gender & 0.233 & -0.108 & 0.245 & -0.089 & $0.383^{* *}$ & 0.093 & 0.237 & 0.053 \\
\hline Age & 0.217 & -0.149 & 0.280 & -0.146 & $0.478 * *$ & $-0.306 *$ & 0.149 & -0.167 \\
\hline Marital status & $-0.344^{*}$ & -0.007 & $-0.397 * *$ & 0.088 & $-0.440 * *$ & 0.060 & $-0.349 * *$ & 0.010 \\
\hline Education & -0.268 & -0.098 & -0.208 & -0.236 & $0.299 *$ & -0.136 & 0.068 & -0.037 \\
\hline Duration of illness & -0.193 & -0.105 & -0.179 & 0.000 & -0.127 & -0.163 & -0.024 & -0.165 \\
\hline Age at onset & $0.354^{*}$ & -0.097 & $0.400 * *$ & -0.163 & $0.549 * *$ & -0.218 & 0.169 & -0.061 \\
\hline Recurrence times & -0.134 & $-0.278^{*}$ & -0.200 & $-0.309 *$ & -0.008 & -0.166 & -0.006 & $-0.314^{*}$ \\
\hline HAMD total score & $-0.543 * *$ & -0.179 & $-0.514 * *$ & $-0.312^{*}$ & $-0.549 * *$ & -0.232 & $-0.464 * *$ & -0.073 \\
\hline HAMA total score & $-0.378 * *$ & -0.239 & -0.283 & $-0.260 *$ & $-0.29 I^{*}$ & -0.173 & $-0.416 * *$ & -0.039 \\
\hline YMRS total score & -0.270 & 0.151 & -0.149 & 0.172 & -0.215 & 0.145 & -0.261 & 0.131 \\
\hline RBANS total score & -0.198 & -0.032 & -0.089 & -0.202 & -0.005 & -0.226 & 0.062 & -0.019 \\
\hline Attention & 0.069 & -0.020 & 0.043 & $-0.268^{*}$ & -0.024 & -0.123 & 0.139 & -0.257 \\
\hline Language & -0.072 & 0.053 & 0.059 & 0.077 & -0.002 & 0.002 & 0.092 & 0.092 \\
\hline Visuospatial & -0.257 & -0.101 & -0.104 & -0.054 & -0.117 & -0.236 & -0.005 & -0.049 \\
\hline Immediate memory & -0.259 & -0.010 & -0.198 & -0.052 & -0.070 & -0.149 & 0.022 & 0.086 \\
\hline Delayed memory & -0.144 & 0.076 & -0.100 & -0.104 & 0.111 & -0.076 & 0.044 & 0.060 \\
\hline \multicolumn{9}{|l|}{ SCT } \\
\hline Sword time & 0.191 & -0.042 & 0.088 & 0.030 & 0.095 & 0.054 & -0.011 & -0.032 \\
\hline Scolour time & 0.012 & 0.238 & 0.006 & 0.259 & -0.031 & 0.145 & -0.184 & 0.106 \\
\hline Dword time & 0.247 & -0.151 & 0.217 & -0.193 & 0.286 & -0.139 & 0.068 & $-0.267^{*}$ \\
\hline Dcolour time & -0.004 & 0.226 & -0.092 & 0.175 & 0.105 & 0.100 & -0.089 & 0.064 \\
\hline
\end{tabular}

Notes: *Correlation is significant at the 0.05 level (2-tailed). **Correlation is significant at the 0.01 level (2-tailed).

Abbreviations: QOL, quality of life; HAMD, Hamilton Depression Rating Scale; HAMA, Hamilton Anxiety Rating Scale; YMRS, Young Mania Rating Scale; RBANS, repeated neuropsychologicalassessment scale; SCT, Stroop Color Test; WHOQOL-BREF, World Health Organization Quality of Life Scale Brief.

The cause of the difference in QOL may be the lack of research directly comparing MDD and BD in terms of symptoms related to depression and anxiety. However, these are speculations; future studies exploring the impact of personality as well as culture on QOL of different mood disorders are warranted. Studies have reported that patients with BD cause more severe functional disability than does MDD. . $^{27,39-41}$

Self-evaluation in terms of overall QOL (total scores in Table 1) yielded lower scores for patients with MDD or BD. The results presented above indicate that individuals with euthymic mood disorders continue to struggle with activities of daily life. It is therefore necessary to improve QOL, especially on physical and psychological domains, among patients with unipolar depression who are currently in remission.

Interestingly, though the RBANS score in patients with euthymic MDD or BD was lower than the individuals without mental diseases, the difference was not significant statistically except for the delayed memory in correlation analyses of the present study. Attention deficits did reduce the psychological satisfaction of patients with $\mathrm{BD}$ in remission, as did Dword time on the SCT for the ENVIR. These results do not completely accord with the views of the previous studies which showed that patients with mood disorders in remission suffer from cognitive deficits which was negatively correlated with QOL. ${ }^{42-44}$ While it was in agreement with the figures in Chinese, explaining the nonsignificant association between cognitive deficits and QOL on account to the distress/protection model of QOL, ${ }^{33,45}$ which considered that QOL is a product of a manual effect of protective and distressing factors. The effects of non-pharmacological treatments such as repetitive transcranial magnetic stimulation (rTMS) may be considered as a promising tool for the enhancement of cognition. ${ }^{46}$

Several limitations have to be addressed. Due caution is necessary to draw conclusions. First, this was a crosssectional descriptive study, as are most investigations of QOL. To be included in the study, patients had HAMD-17 score $\leq 7$ and YMRS score $\leq 6$ at intake. However, the 
Table 3 The multivariate regression analysis of influential factors on the life qualities of patients with MDD patients

\begin{tabular}{|c|c|c|c|c|c|}
\hline Dependent variable & $\begin{array}{l}\text { Independent } \\
\text { variable }\end{array}$ & Coefficient & $\begin{array}{l}\text { Standardized } \\
\text { coefficient }\end{array}$ & $\mathbf{t}$ & $p$ \\
\hline \multirow[t]{4}{*}{ Physical $\left(R^{\prime}=0.428, F=8.044, P<0.00 I\right)$} & Marital status & 0.863 & -0.222 & -1.798 & 0.079 \\
\hline & Age at onset & 0.038 & 0.186 & 1.477 & 0.147 \\
\hline & HAMD total** & $-0.40 \mathrm{I}$ & -0.404 & -3.075 & 0.004 \\
\hline & HAMA total & -0.089 & -0.188 & -1.459 & 0.152 \\
\hline \multirow[t]{3}{*}{ Psychological $\left(R^{\prime}=0.42 I, F=10.650 P<0.00 I\right)$} & marital status* & -1.092 & -0.272 & -2.221 & 0.032 \\
\hline & Age at onset & 0.045 & 0.213 & 1.708 & 0.095 \\
\hline & HAMD total** & -0.458 & -0.448 & -3.824 & $\leq 0.001$ \\
\hline \multirow[t]{7}{*}{ Social $\left(R^{\prime}=0.600, F=8.583, P<0.001\right)$} & Gender & 1.058 & 0.202 & $\mathrm{I} .745$ & 0.089 \\
\hline & Age & 0.043 & 0.165 & 1.020 & 0.314 \\
\hline & Marital status & -0.936 & -0.207 & -1.895 & 0.065 \\
\hline & Education & -0.036 & -0.047 & -0.427 & 0.672 \\
\hline & Age at onset & 0.046 & 0.195 & 1.152 & 0.256 \\
\hline & HAMD total** & -0.478 & -0.415 & -3.484 & 0.001 \\
\hline & HAMA total & -0.006 & -0.011 & -0.085 & 0.932 \\
\hline \multirow[t]{3}{*}{ Environmental $\left(R^{\prime}=0.378, F=8.920, P<0.00 I\right)$} & marital status $* *$ & -1.226 & -0.329 & -2.758 & 0.008 \\
\hline & HAMD tota* & -0.304 & -0.320 & -2.411 & 0.020 \\
\hline & HAMA total & -0.120 & -0.0265 & -2.004 & 0.051 \\
\hline
\end{tabular}

Notes: *Analysis is significant at the 0.05 level (2-tailed). **Analysis is significant at the 0.01 level (2-tailed).

Abbreviations: MDD, major depressive disorder; HAMA, Hamilton Anxiety Rating Scale; HAMD, Hamilton Depression Rating Scale.

Table 4 The multivariate regression analysis of influential factors on the life qualities of patients with BD patients

\begin{tabular}{|c|c|c|c|c|c|}
\hline Dependent variable & Independent variable & Coefficient & Standardized coefficient & $\mathbf{t}$ & $p$ \\
\hline Physical $\left(R^{\prime}=0.027, F=1.590, P=0.212\right)$ & Recurrence times & -0.030 & -0.165 & -1.261 & 0.212 \\
\hline \multirow[t]{4}{*}{ Psychological $\left(R^{\prime}=0.236, F=4.025, P=0.006\right)$} & Recurrence times & -0.034 & -0.184 & -1.504 & 0.139 \\
\hline & HAMD total & -0.308 & -0.257 & -1.542 & 0.129 \\
\hline & HAMA total & -0.129 & -0.155 & -0.934 & 0.354 \\
\hline & Attention* & -0.042 & -0.250 & -2.054 & 0.045 \\
\hline Social $\left(R^{\prime}=0.094, F=5.893, P=0.018\right)$ & Age* & -0.078 & -0.306 & -2.428 & 0.018 \\
\hline \multirow[t]{2}{*}{ Environmental $\left(R^{\prime}=0.072, F=2.059, P=0.138\right)$} & Recurrence times & -0.005 & -0.028 & -0.188 & $0.85 I$ \\
\hline & Dword time & -0.077 & -0.256 & -1.752 & 0.086 \\
\hline
\end{tabular}

Notes: *Analysis is significant at the 0.05 level (2-tailed).

Abbreviations: BD, bipolar disorder; HAMA, Hamilton Anxiety Rating Scale; HAMD, Hamilton Depression Rating Scale.

possibility of fluctuations in subsyndromal symptoms during the euthymic period may not be excluded. The subsyndromal symptoms may impact on the WHOQOLBREF domain scores differently. Because both the MDD and $\mathrm{BD}$ are chronic illness with not only multiple episodes but also fluctuating residual symptoms, it will be vital to conduct respective survey to assess QOL over extended periods. Second, the sample size is relatively small, which might have decreased statistical power. A larger sample size would have allowed the use of more complex regression models to explore the impact of a wider range of clinical and psychological factors on QOL. Additionally, we recruited the patients with euthymic BD I only, and the data between subtypes of BD were insufficient. Third, the data of socio-demographic, clinical information were gathered by recalling assessment and may, thus, be distorted by memory. The results of cognitive testing were assessed by a single rater. Different aspects of the assessment should have been implemented by different researchers blind to each other ideally. 


\section{Conclusion}

In summary, poorer satisfaction with QOL does persist in patients with MDD and BD even in remission, suggesting that keeping a finger on the pulse of developments in QOL enhancement will likely be important for clinicians treating either for MDD or BD. During remission period, the QOL of patients with MDD is significantly poorer than that of BD, which was correlated with depressive and anxiety symptoms, suggesting that syndrome recovery does necessarily result in QOL comparable to that of healthy individuals. It is indicated the importance of QOL assessment on mood disorder patients in both clinical practice and research. Efforts to treat residual symptoms and cognitive impairment may enhance QOL in both MDD and BD patients.

More studies with larger sample sizes are needed to examine the effect of demographic and clinical variables that could account for poor QOL in euthymic patients with mood disorders and compare the relationship and difference of QOL and cognitive function between MDD and BD. Additional studies will be necessary to confirm the findings reported above. Such efforts may contribute to the identification of patients who may require interventions aimed at management of QOL and rehabilitation of cognitive function. In the future, research into this vital area and as clinical trials are designed and new instruments developed. We suggest an emphasis not just on symptom severity and functional status but also on QOL as measured by the patient's selfreported level of satisfaction and perceptions. Maybe finding the inherent laws of QOL and mood disorders would provide us a new way to identify the recurrence of mood disorders.

\section{Acknowledgments}

This study was supported by Beijing Science and Technology Commission (D121100005012002), Beijing Municipal Administration of Hospital Youth Program (QML20171901) and Beijing Municipal Administration of Hospitals Clinical Medicine Development of Special Funding (ZYLX201807). No investigator benefited from participating in the study.

\section{Disclosure}

The authors report no conflicts of interest in this work.

\section{References}

1. Dave VA. Mood disorder. Paripex Indian J Res. 2013;2(2): 259-261.
2. GROUP TW. Development of the World Health Organization WHOQOL-BREF quality of life. 1998.

3. Kurtz MM, Bronfeld M, Rose J. Cognitive and social cognitive predictors of change in objective versus subjective quality-of-life in rehabilitation for schizophrenia. Psychiatry Res. 2012;200(2-3):102-107. doi:10.1016/j.psychres.2012.06.025

4. Cotrena C, Branco LD, Kochhann R, Shansis FM, Fonseca RP. Quality of life, functioning and cognition in bipolar disorder and major depression: a latent profile analysis. Psychiatry Res. 2016;241:289-296. doi:10.1016/j.psychres.2016.04.102

5. IsHak WW, Greenberg JM, Balayan K, et al. Quality of life: the ultimate outcome measure of interventions in major depressive disorder. Harv Rev Psychiatry. 2011;19(5):229-239. doi:10.3109/ 10673229.2011.614099

6. Cramer VT, Orgersen S,EK. Mood disorders and quality of life. A community study. Nord J Psychiatry. 2010;64(1):58-62. doi:10.3109/08039480903287565

7. Michalak EE, Yatham LN, Lam RW. Quality of life in bipolar disorder: a review of the literature. Health Qual Life Outcomes. 2005;3 (1):72. doi:10.1186/1477-7525-3-72

8. Jha MK, Greer TL, Grannemann BD, Carmody T, Rush AJ, Trivedi MH. Early normalization of quality of life predicts later remission in depression: findings from the CO-MED trial. $J$ Affect Disord. 2016;206:17-22. doi:10.1016/j.jad.2016.07.012

9. Thunedborg K, Black CH, Bech P. Beyond the Hamilton depression scores in long-term treatment of manic-melancholic patients: prediction of recurrence of depression by quality of life measurements. Psychother Psychosom. 1995;64(3-4):131-140. doi:10.1159/000289002

10. Atkinson M, Zibin S, Chuang H. Characterizing quality of life among patients with chronic mental illness: a critical examination of the self-report methodology. Am J Psychiatry. 1997;154(1):99-105. doi:10.1176/ajp.154.1.99

11. Cooke RG, Robb JC, Young LT, Joffe RT. Well-being and functioning in patients with bipolar disorder assessed using the MOS 20-ITEM short form (SF-20). J Affect Disord. 1996;39(2):93-97.

12. Michalak EE, Yatham LN, Wan DD, Lam RW. Perceived quality of life in patients with bipolar disorder. Does group psychoeducation have an impact? Can J Psych Rev Can Psychiatr. 2005;50(2):95-100. doi:10.1177/070674370505000204

13. Sierra P, Livianos L, Rujo L. Quality of life for patients with bipolar disorder relationship with clinical and demographic variables. Bipolar Disord. 2005;7(2):159-165. doi:10.1111/j.1399-5618.2005.00186.x

14. IsHak WW, Mirocha J, James D, et al. Quality of life in major depressive disorder before/after multiple steps of treatment and one-year follow-up. Acta Psychiatr Scand. 2015;131(1):51-60. doi:10.1111/acps. 12301

15. Saragoussi D, Christensen MC, Hammer-Helmich L, Rive B, Touya M, Haro JM. Long-term follow-up on health-related quality of life in major depressive disorder: a 2-year European cohort study. Neuropsychiatr Dis Treat. 2018;14:1339-1350. doi:10.2147/NDT.S159276

16. First MB, Spitzer RL, Gibbon M, Williams JB Structured clinical interview for DSM-IV-TR axis I disorders, research version, patient edition. (SCID-I/P) Biometrics Research Department, New York State Psychiatric Institute USA 2002.

17. Li T, Zhou R, Hu J, et al. Translation. Revision. Structured Clinical Interview for DSM-IV-TR Axis I Disorders-Patient Edition (SCID-I/ P). Psychiatry Research Center, Beijing Huilongguan Hsopital China 2008.

18. Hamilton M. A rating scale for depression. $J$ Neurol Neurosurg Psychiatry. 1960;23:56-62. doi:10.1136/jnnp.23.1.56

19. Hamilton M. The assessment of anxiety states by rating. $B r J$ Med Psychol. 1959;32(1):50-55.

20. Young RC, Biggs JT, Ziegler VE, Meyer DA. A rating scale for mania: reliability, validity and sensitivity. $B r J$ Psychiatry. 1978;133 (5):429-435. 
21. Hao Y, Fang J. The introduce and usage of World Health Organization Quality of Life Scale (WHOQOL) instrument in chinese. Mod Rehabil (Chinese). 2000;4(8):1127-1129.

22. Randolph C. Repeatable Battery for the Assessment of Neuropsychological Status (RBANS). Psychological Corporation San Antonio, TX; 1998.

23. Zhang BH, Tan YL, Zhang WF, et al. Repeatable battery for the assessment of neuropsychological status as a screening test in chinese: reliability and validity. Chin Ment Health J. 2008;22(18):865-869.

24. Stroop JR. Studies of interference in serial verbal reactions. $J$ Exp Psychol. 1935;18(6):643. doi:10.1037/h0054651

25. Hochberg Y. A sharper Bonferroni procedure for multiple tests of significance. Biometrika. 1988;75(4):800-802. doi:10.1093/biomet/ 75.4.800

26. Angermeyer MC, Holzinger A, Matschinger H. Depression and quality of life: results of a follow-up study. Int J Soc Psychiatry. 2002;48 (3):189-199. doi:10.1177/002076402128783235

27. van der Voort TYG, Seldenrijk A, van Meijel B, et al. Functional versus syndromal recovery in patients with major depressive disorder and bipolar disorder. J Clin Psychiatry. 2015;76:e809-e814. doi:10.4088/JCP.14m09548

28. Rubio JM, Olfson M, Villegas L, Perez-Fuentes G, Wang S, Blanco C. Quality of life following remission of mental disorders: findings from the National Epidemiologic Survey on Alcohol and Related Conditions. J Clin Psychiatry. 2013;74(5):e445-e450. doi:10.4088/JCP.12m08269

29. Zimmerman M, Martinez JA, Attiullah N, et al. Why do some depressed outpatients who are in remission according to the Hamilton Depression Rating Scale not consider themselves to be in remission? J Clin Psychiatry. 2012;73(6):790-795. doi:10.4088/JCP.11m07203

30. Brissos S, Dias VV, Carita AI, Martinez-Arán A. Quality of life in bipolar type I disorder and schizophrenia in remission: clinical and neurocognitive correlates. Psychiatry Res. 2008;160(1):55-62. doi:10.1016/j.psychres.2007.04.010

31. Maina G, Albert U, Bellodi L. Health-related quality of life in euthymic bipolar disorder patients differences between bipolar I and II subtypes. J Clin Psychiatry. 2008;68(2):207-212. doi:10.4088/JCP.v68n0205

32. Costa J, García-Blanco A, Cañada Y, et al. Are affective temperaments determinants of quality of life in euthymic patients with bipolar disorder? $J$ Affect Disord. 2018;230:101-107. doi:10.1016/j.jad.2018.01.007

33. Xiang YT, Li LJ, Zhou JJ, et al. Quality of life of patients with euthymic bipolar disorder and its associations with demographic and clinical characteristics, psychopathology, and cognitive deficits. Perspect Psychiatr Care. 2014;50(1):44-50. doi:10.1111/ppc.12024

34. Murray G. The quality of life construct in bipolar disorder research and practice past present and possible futures. Bipolar Disord. 2012;14(8):793-796. doi:10.1111/bdi.12016
35. Morton EMEE, Murray G. What does quality of life refer to in bipolar disorders research? A systematic review of the construct's definition, usage and measuremen. J Affect Disord. 2017;212:128-137. doi:10.1016/j.jad.2017.01.026

36. Morton E, Murray G, Michalak EE, et al. Quality of life in bipolar disorder: towards a dynamic understanding. Psychol Med. 2018; 48 (7): 1111-1118.

37. Gutiérrez-Rojas L, Gurpegui M, Ayuso-Mateos JL. Quality of life in bipolar disorder patients a comparison with a general population sample. Bipolar Disord. 2008;10(5):625-634. doi:10.1111/j.13995618.2008.00604.x

38. Judd LL, Akiskal HS, Maser JD, Zeller PJ. Major depressive disorder a prospective study of residual subthreshold depressive symptoms as predictor of rapid relapse. J Affect Disord. 1998;50:97-108.

39. Shippee ND, Shah ND, Williams MD, Moriarty JP, Frye MA, Ziegenfuss JY. Differences in demographic composition and in work, social, and functional limitations among the populations with unipolar depression and bipolar disorder: results from a nationally representative sample. Health Qual Life Outcomes. 2011;9(1). doi:10.1186/1477-7525-9-40

40. Goldberg JF, Harrow M. Subjective life satisfaction and objective functional outcome in bipolar and unipolar mood disorders: a longitudinal analysis. $J$ Affect Disord. 2005;89(1-3):79-89. doi:10.1016/j.jad.2005.08.008

41. Judd LL, Schettler PJ, Solomon DA, et al. Psychosocial disability and work role function compared across the long-term course of bipolar I, bipolar II and unipolar major depressive disorders. J Affect Disord. 2008;108(1-2):49-58. doi:10.1016/j.jad.2007.06.014

42. PDH. Mood symptoms cognition and everyday functioning in major depression, bipolar disorder, and schizophrenia. Innov Clin Neurosci. 2011;8(10):14-18.

43. Evans VC, Chan SS, Iverson GL, Bond DJ. Systematic review of neurocognition and occupational functioning in major depressive disorder. Neuropsychiatry. 2013;3(1):97-105. doi:10.2217/npy.13.3

44. Martino DJ, Strejilevich SA, Marengo E, Ibanez A, Scapola M, Igoa A. Toward the identification of neurocognitive subtypes in euthymic patients with bipolar disorder. $J$ Affect Disord. 2014;167:118-124. doi:10.1016/j.jad.2014.05.059

45. Voruganti L, Heslegrave R, Awad AG. Quality of life measurement in schizophrenia: reconciling the quest for subjectivity with the question of reliability. Psychol Med. 1998;28(1):165-172.

46. Serafini G, Pompili M, Belvederi Murri M, et al. The effects of repetitive transcranial magnetic stimulation on cognitive performance in treatment-resistant depression. A systematic review. Neuropsychobiology. 2015;71(3):125-139. doi:10.1159/000381351
Neuropsychiatric Disease and Treatment

\section{Publish your work in this journal}

Neuropsychiatric Disease and Treatment is an international, peerreviewed journal of clinical therapeutics and pharmacology focusing on concise rapid reporting of clinical or pre-clinical studies on a range of neuropsychiatric and neurological disorders. This journal is indexed on PubMed Central, the 'PsycINFO' database and CAS, and is the official journal of The International Neuropsychiatric Association (INA). The manuscript management system is completely online and includes a very quick and fair peer-review system, which is all easy to use. Visit http://www.dovepress.com/testimonials.php to read real quotes from published authors. 